Annuaire suisse de politique de développement

24-1 | 2005

Faits et statistiques 2005

\title{
C. Caractéristiques des relations entre la Suisse et les pays en développement
}

Gérard Perroulaz et Xavier Tschumi Canosa

\section{OpenEdition}

Édition électronique

URL : http://journals.openedition.org/aspd/439

DOI : 10.4000/aspd.439

ISSN : 1663-9669

Éditeur

Institut de hautes études internationales et du développement

\section{Édition imprimée}

Date de publication : 1 avril 2005

Pagination : 230-244

ISSN : 1660-5934

\section{Référence électronique}

Gérard Perroulaz et Xavier Tschumi Canosa, «C. Caractéristiques des relations entre la Suisse et les pays en développement », Annuaire suisse de politique de développement [En ligne], 24-1 | 2005, mis en ligne le 05 mars 2010, consulté le 08 septembre 2020. URL : http://journals.openedition.org/aspd/439 ; DOI : https://doi.org/10.4000/aspd.439 


\section{Caractéristiques des relations entre la Suisse et les pays en développement}

\section{C.1. Faits saillants}

Les tableaux introductifs 1 à 3 donnent la vue d'ensemble des relations de la Suisse avec tous les pays en développement et les pays en transition (voir la liste des pays selon la classification du CAD à la fin de cet Annuaire). Ils permettent de faire ressortir quelques caractéristiques de ces relations :

$\square$ Les apports du secteur privé sont dans l'ensemble beaucoup plus importants que les apports d'aide publique et privée. Si, en 2003, l'aide publique bilatérale aux pays en développement s'élève à 1271 millions de francs et l'aide des $\mathrm{ONG}$ à 377 millions de francs, les investissements directs à l'étranger atteignent 2,8 milliards de francs. Les relations économiques avec les pays en transition sont encore plus fortes pour cette même année: les pays en transition ont reçu 90,9 millions de francs d'APD et 15,4 millions de francs d'aide des $\mathrm{ONG}$, et les investissements se sont élevés à 1,6 milliard de francs.

- La répartition géographique est très différente entre l'aide publique et les flux d'investissements privés: l'APD et l'aide des ONG sont essentiellement destinées aux pays à faible revenu, tandis que les investissements se dirigent surtout vers les pays à revenu moyen et les pays d'Europe centrale, et les exportations vers les pays à revenu moyen, les pays d'Europe centrale et orientale et les pays en développement avancés.

口 La situation des pays africains est intéressante à relever: ils reçoivent $27 \%$ de l'aide publique au développement bilatérale et le tiers de l'aide des ONG, mais ne représentent que $20 \%$ des importations de la Suisse en provenance des pays en développement et $12 \%$ des exportations; les investissements nets sont même négatifs, ce qui représente un désinvestissement.

- La situation des 49 pays les moins avancés (PMA) est semblable: alors que les PMA ont reçu en $200327 \%$ de l'APD totale et $40 \%$ de l'aide des ONG, seul $1,9 \%$ des importations en provenance des pays en développement (PED) émane de ces pays et les flux nets d'investissements n'ont constitué que $0,8 \%$ des investissements totaux vers les PED. Les PMA ne représentent que $0,12 \%$ des importations totales de la Suisse.

$\square$ Les flux financiers nets entre les pays en développement et la Suisse sont généralement en faveur de cette dernière. Si l'on prend en considération uniquement les flux d'aide publique et privée, les investissements et le solde commercial avec certaines régions en développement, on remarque que les flux de ces régions vers la Suisse sont supérieurs aux flux d'aide de la Suisse vers ces régions. Ces données sont partielles, car on ne tient pas compte de certains flux, difficiles à répartir par catégories de pays, comme les flux bancaires (service et remboursement de la dette, placements nets dans les banques suisses) ou les versements des migrants en Suisse à leurs familles. 
- L'aide publique bilatérale de la Confédération et l'aide des ONG suisses (aide financée par des fonds privés) ont une répartition géographique assez semblable, avec cependant, pour l'aide des ONG, une part plus importante destinée aux pays en développement d'Asie, d'Afrique et d'Amérique latine.

\section{C.1.1. Place des pays en développement et des pays en transition dans le commerce extérieur de la Suisse (tableaux de la section B)}

Les importations totales de la Suisse s'élevaient à 129,7 milliards de francs en 2003. Si l'on applique la classification des pays établie par le CAD, les importations de la Suisse en provenance des pays en développement ont atteint 8,2 milliards de francs (soit 6,3\% des importations totales) et les importations en provenance des pays en transition 7,5 milliards de francs. Seuls $12,1 \%$ des importations proviennent des pays en développement et des pays en transition, alors que $23 \%$ des exportations vont vers ces pays.

La Suisse a le plus souvent une balance commerciale excédentaire avec les pays en développement et les pays en transition, alors que la balance commerciale est généralement déficitaire avec les pays industrialisés (voir les trois dernières colonnes des tableaux de la section B). Par exemple, en 2003, la Suisse a importé pour 41,9 milliards de francs de produits en provenance d'Allemagne et exporté pour 28,2 milliards de francs vers ce pays (soit un déficit commercial de 13,7 milliards de francs). L'excédent commercial avec l'ensemble des pays en développement s'est élevé en 2003 à 7,1 milliards de francs; l'excédent commercial avec les pays en développement avancés a atteint quant à lui 6,9 milliards de francs, et celui avec les pays d'Europe centrale et orientale 1,4 milliard de francs.

\section{Graphique 1: Pays en développement et en transition \\ avec lesquels l'excédent commercial est le plus important, 2003}

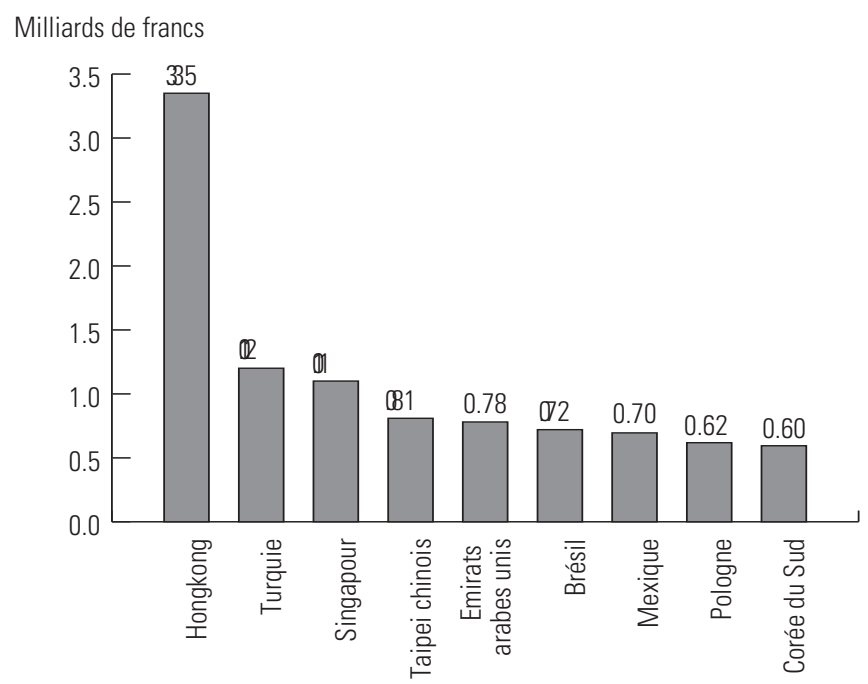

Source: Direction générale des douanes, Berne, Commerce extérieur de la Suisse, statistique selon les marchandises et les pays. 
Le cas de la Chine est intéressant à relever, car la Suisse importait ces dernières années beaucoup plus qu'elle n'exportait vers ce pays, avec un solde commercial négatif allant en diminuant. Le solde commercial est devenu légèrement positif en 2003.

\section{C.2. Commerce extérieur de la Suisse}

\section{C.2.1. Vue d'ensemble}

Schéma 1: Commerce de la Suisse avec les pays en développement et en transition, 2003 (en millions de francs)

$M=$ importations de la Suisse

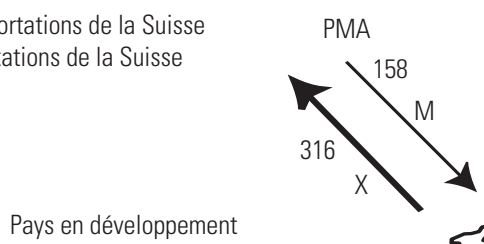

Pays en développement à revenu moyen

= exportations de la Suisse
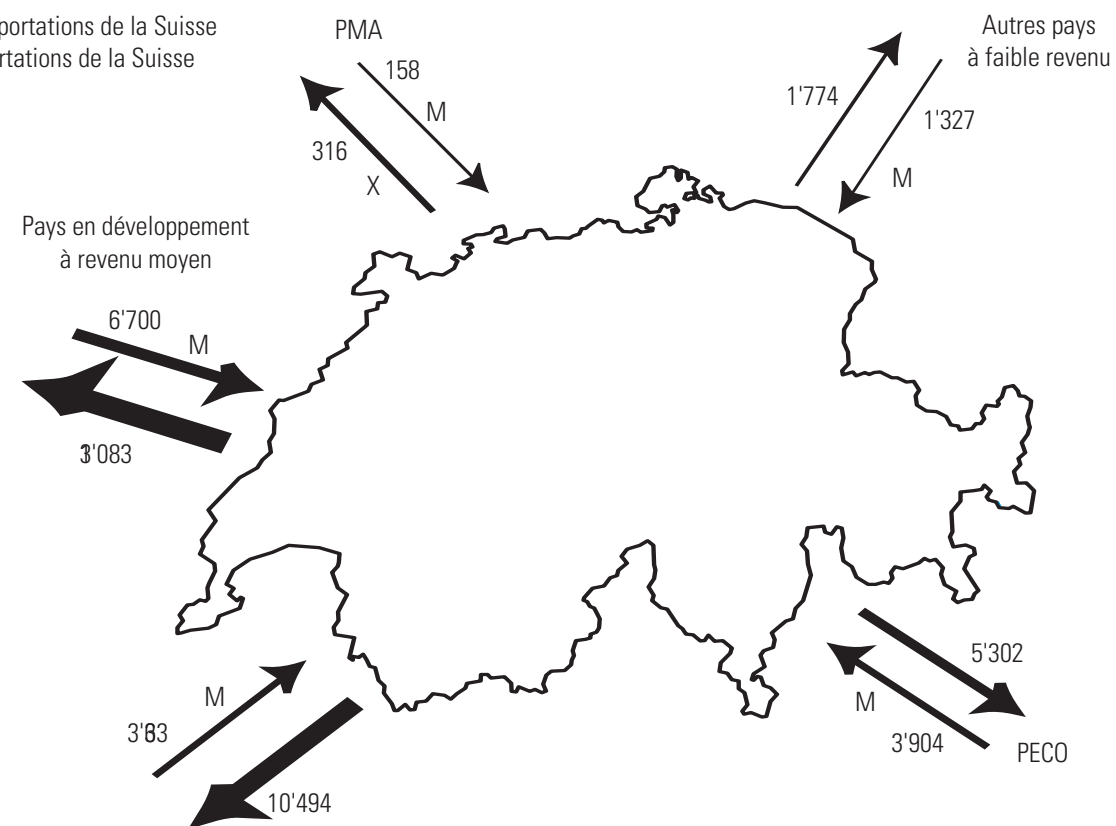

Pays en développement

plus avancés

Source: adaptée de: Aus Fehlern lernen? Die Schweiz und die Dritte Welt, Richard Gerster, Edition Ex Libris, Zurich, p. 65; Direction générale des douanes, Statistique du commerce extérieur de la Suisse, 2004, pp. 869-871 pour les chiffres actualisés.

La Suisse entretient des relations commerciales surtout avec les pays voisins et les pays industrialisés; $58 \%$ de ses importations proviennent des pays limitrophes et $44,5 \%$ de ses exportations leur sont destinés. Comme on le voit dans le tableau 4, seuls 5 pays en développement ou en transition figurent parmi les 20 principaux pays fournisseurs de la Suisse et 8 pays figurent dans les 20 principaux pays clients de la Suisse. 
Tableau 4: Les 20 premiers partenaires commerciaux de la Suisse, 2003

\begin{tabular}{|c|c|c|c|c|c|c|c|}
\hline \multicolumn{4}{|c|}{ Principaux fournisseurs } & \multicolumn{4}{|c|}{ Principaux clients } \\
\hline \multicolumn{4}{|c|}{ Importations } & \multicolumn{3}{|c|}{ Exportations } & \\
\hline Pays $^{a}$ & Mio fr. & $\%$ & $\sum \%$ & Pays $^{a}$ & Mio fr. & $\%$ & $\sum \%$ \\
\hline 1. Allemagne féd. & $41^{\prime} 895.5$ & 32.3 & 32.3 & 1. Allemagne féd. & $28^{\prime} 217.3$ & 20.8 & 20.8 \\
\hline 2. France & $13^{\prime} 983.3$ & 10.8 & 43.1 & 2. USA & $14^{\prime} 943.4$ & 11.0 & 31.9 \\
\hline 3. Italie & $13^{\prime} 854.0$ & 10.7 & 53.7 & 3. France & $11^{\prime} 815.1$ & 8.7 & 40.6 \\
\hline 4. USA & $6^{\prime} 634.8$ & 5.1 & 58.9 & 4. Italie & $11^{\prime} 186.0$ & 8.3 & 48.9 \\
\hline 5. Pays-Bas & $66^{\prime} 475.1$ & 5.0 & 63.9 & 5. Grande-Bretagne & $6 \prime 582.1$ & 4.9 & 53.7 \\
\hline 6. Autriche & $5^{\prime} 487.4$ & 4.2 & 68.1 & 6. Japon & 5'405.7 & 4.0 & 57.7 \\
\hline 7. Grande-Bretagne & $5^{\prime} 260.2$ & 4.1 & 72.1 & 7. Espagne & $4^{\prime} 744.0$ & 3.5 & 61.2 \\
\hline 8. Irlande & 4'794.1 & 3.7 & 75.8 & 8. Autriche & 4'469.4 & 3.3 & 64.5 \\
\hline 9. Belgique & $3 \prime 943.0$ & 3.0 & 78.9 & 9. Pays-Bas & $4^{\prime} 402.1$ & 3.3 & 67.8 \\
\hline 10. Espagne & $3^{\prime} 040.5$ & 2.3 & 81.2 & 10. Hongkong & $4^{\prime} 002.2$ & 3.0 & 70.7 \\
\hline 11. Japon & 2728.5 & 2.1 & 83.3 & 11. Belgique & $2^{\prime} 649.4$ & 2.0 & 72.7 \\
\hline 12. Chine & $2^{\prime} 413.9$ & 1.9 & 85.2 & 12. Chine & $2^{\prime} 460.0$ & 1.8 & 74.5 \\
\hline 13. Suède & 1'660.8 & 1.3 & 86.5 & 13. Turquie & 1'650.2 & 1.2 & 75.7 \\
\hline 14. Russie & 1'175.6 & 0.9 & 87.4 & 14. Suède & 1'620.2 & 1.2 & 76.9 \\
\hline 15. Danemark & 1'057.8 & 0.8 & 88.2 & 15. Canada & 1'428.9 & 1.1 & 78.0 \\
\hline 16. Rep. tchèque & 882.8 & 0.7 & 88.9 & 16. Singapour & 1'358.7 & 1.0 & 79.0 \\
\hline 17. Afrique du Sud & 862.2 & 0.7 & 89.5 & 17. Taïwan & 1'329.7 & 1.0 & 80.0 \\
\hline 18. Finlande & 797.3 & 0.6 & 90.1 & 18. Brésil & 1'163.6 & 0.9 & 80.8 \\
\hline 19. Libye & 755.9 & 0.6 & 90.7 & 19. Arabie saoudite & e 1'123.7 & 0.8 & 81.6 \\
\hline 20. Hongrie & 711.2 & 0.5 & 91.3 & 20. Pologne & 1'122.9 & 0.8 & 82.5 \\
\hline Autres pays & $11^{\prime} 328.9$ & 8.7 & 100.0 & Autres pays & $23^{\prime} 730.4$ & 17.5 & 100.0 \\
\hline Total & $129^{\prime} 742.8$ & & 100.0 & Total & $1355^{\prime} 405.0$ & & 100.0 \\
\hline
\end{tabular}

Source: Direction générale des douanes, Commerce extérieur de la Suisse, statistique selon les marchandises et les pays, Berne, 2004, pp. 869-871.

a En gras, pays compris dans la liste des pays en développement (partie I de la liste du CAD) ou des pays en transition (partie ॥ de la liste du CAD).

Nous adoptons dès cette année une répartition géographique du commerce extérieur sur la base de la classification des pays établie par le CAD. Les tableaux 5 et 6 ne sont donc pas comparables avec les tableaux de la partie «Commerce» des années précédentes dans lesquels nous appliquions la classification des pays établie par la CNUCED. Le commerce avec tous les pays en développement et les pays en transition est détaillé dans les trois dernières colonnes des tableaux 1 à 3 de la section $\mathrm{B}$ de la présente partie statistique. 


\section{C.2.2. Pays en développement et pays en transition}

Les tableaux 5 et 6 montrent les 15 pays principaux partenaires commerciaux, respectivement dans le groupe des pays en développement et dans le groupe des pays en transition.

Tableau 5: Les 15 premiers partenaires commerciaux de la Suisse dans les pays en développement ${ }^{\mathrm{a}}, 2003$

\begin{tabular}{|c|c|c|c|c|c|c|c|}
\hline \multicolumn{4}{|c|}{ Principaux fournisseurs } & \multicolumn{4}{|c|}{ Principaux clients } \\
\hline \multicolumn{4}{|c|}{ Importations } & \multicolumn{4}{|c|}{ Exportations } \\
\hline Pays & Mio fr. & $\%^{b}$ & $\%{ }^{c}$ & Pays & Mio fr. & $\%^{b}$ & $\%^{c}$ \\
\hline 1. Chine & $2 ' 413.9$ & 29.4 & 1.86 & 1. Chine & $2^{\prime} 460.0$ & 16.1 & 1.82 \\
\hline 2. Afrique du Sud & 862.2 & 10.5 & 0.66 & 2. Turquie & 1'650.2 & 10.8 & 1.22 \\
\hline 3. Thaïlande & 608.1 & 7.4 & 0.47 & 3. Brésil & 1'163.6 & 7.6 & 0.86 \\
\hline 4. Inde & 500.2 & 6.1 & 0.39 & 4. Arabie saoudite & 1'123.7 & 7.4 & 0.83 \\
\hline 5. Brésil & 443.9 & 5.4 & 0.34 & 5. Mexique & 914.0 & 6.0 & 0.68 \\
\hline 6. Turquie & 432.6 & 5.3 & 0.33 & 6. Thaïlande & 777.6 & 5.1 & 0.57 \\
\hline 7. Nigeria & 351.8 & 4.3 & 0.27 & 7. Inde & 741.6 & 4.9 & 0.55 \\
\hline 8. Mexique & 218.6 & 2.7 & 0.17 & 8. Iran & 541.5 & 3.5 & 0.40 \\
\hline 9. Liban & 190.8 & 2.3 & 0.15 & 9. Afrique du Sud & 523.0 & 3.4 & 0.39 \\
\hline 10. Malaisie & 174.7 & 2.1 & 0.13 & 10. Malaisie & 449.4 & 2.9 & 0.33 \\
\hline 11. Arabie saoudite & 173.8 & 2.1 & 0.13 & 11. Egypte & 415.1 & 2.7 & 0.31 \\
\hline 12. Iran & 170.5 & 2.1 & 0.13 & 12. Indonésie & 289.4 & 1.9 & 0.21 \\
\hline 13. Indonésie & 167.1 & 2.0 & 0.13 & 13. Argentine & 248.8 & 1.6 & 0.18 \\
\hline 14. Vietnam & 151.3 & 1.8 & 0.12 & 14. Pakistan & 245.5 & 1.6 & 0.18 \\
\hline 15. Algérie & 128.5 & 1.6 & 0.10 & 15. Croatie & 213.7 & 1.4 & 0.16 \\
\hline Autres pays en dév. & 1'209.3 & 14.8 & 0.93 & Autres pays en dév. & $3^{\prime} 531.2$ & 23.1 & 2.61 \\
\hline Total & 8'197.3 & 100.0 & 6.32 & Total & $15^{\prime} 288.3$ & 100.0 & 11.29 \\
\hline
\end{tabular}

Source: Direction générale des douanes, Statistique du commerce extérieur de la Suisse, 2004, pp. 869-871.

a Pays en développement selon la partie I de la liste du CAD (reproduite à la fin de l'Annuaire).

${ }^{b}$ Pourcentage du pays dans le total des importations/exportations des pays en développement.

c Pourcentage du pays dans le total des importations/exportations de tous les pays partenaires de la Suisse. 
Tableau 6: Les 15 premiers partenaires commerciaux de la Suisse dans les pays en transition ${ }^{\mathrm{a}}, 2003$

\begin{tabular}{|c|c|c|c|c|c|c|c|c|}
\hline \multicolumn{4}{|c|}{ Principaux fournisseurs } & \multicolumn{5}{|c|}{ Principaux clients } \\
\hline \multicolumn{4}{|c|}{ Importations } & \multicolumn{5}{|c|}{ Exportations } \\
\hline Pays & Mio fr. & $\%^{\mathrm{b}}$ & $\%^{c}$ & & Pays & Mio fr. & $\%^{\mathrm{b}}$ & $\% c$ \\
\hline 1. Russie & 1'175.6 & 15.6 & 0.91 & 1. & Hongkong & $4^{\prime} 002.2$ & 25.3 & 2.96 \\
\hline 2. Rép. tchèque & 882.8 & 11.7 & 0.68 & 2. & Singapour & 1'358.7 & 8.6 & 1.00 \\
\hline 3. Libye & 755.9 & 10.0 & 0.58 & 3. & Taïwan & 1'329.7 & 8.4 & 0.98 \\
\hline 4. Hongrie & 711.2 & 9.4 & 0.55 & 4. & Pologne & 1'122.9 & 7.1 & 0.83 \\
\hline 5. Hongkong & 648.7 & 8.6 & 0.50 & 5. & Corée du Sud & 1'101.6 & 7.0 & 0.81 \\
\hline 6. Taïwan & 521.0 & 6.9 & 0.40 & 6. & Russie & 1'064.7 & 6.7 & 0.79 \\
\hline 7. Corée du Sud & 507.7 & 6.7 & 0.39 & 7. & Rép. tchèque & 1'032.4 & 6.5 & 0.76 \\
\hline 8. Pologne & 504.6 & 6.7 & 0.39 & 8. & Emirats arabes unis & 908.5 & 5.8 & 0.67 \\
\hline 9. Israël & 370.9 & 4.9 & 0.29 & & Hongrie & 846.8 & 5.4 & 0.63 \\
\hline 10. Slovaquie & 306.6 & 4.1 & 0.24 & 10. & Israël & 626.6 & 4.0 & 0.46 \\
\hline 11. Singapour & 228.5 & 3.0 & 0.18 & 11. & Roumanie & 305.2 & 1.9 & 0.23 \\
\hline 12. Slovénie & 173.0 & 2.3 & 0.13 & 12. & Slovénie & 292.8 & 1.9 & 0.22 \\
\hline 13. Vierges, îles & 160.2 & 2.1 & 0.12 & 13. & Slovaquie & 283.4 & 1.8 & 0.21 \\
\hline 14. Roumanie & 142.2 & 1.9 & 0.11 & 14. & Ukraine & 199.1 & 1.3 & 0.15 \\
\hline 15. Emirats arabes unis & 128.3 & 1.7 & 0.10 & 15. & Libye & 122.3 & 0.8 & 0.09 \\
\hline $\begin{array}{l}\text { Autres pays } \\
\text { en transition }\end{array}$ & 319.5 & 4.2 & 0.25 & & $\begin{array}{l}\text { Autres pays } \\
\text { en transition }\end{array}$ & 1'198.8 & 7.6 & 0.89 \\
\hline Total & 7'536.7 & 100.0 & 5.81 & & Total & '795.7 & 100.0 & 11.67 \\
\hline
\end{tabular}

Source: Direction générale des douanes, Statistique du commerce extérieur de la Suisse, 2004, pp. 869-871.

a Pays en développement plus avancés et pays d'Europe centrale et orientale selon la partie II de la liste du CAD (reproduite à la fin de l'Annuaire).

b Pourcentage du pays dans le total des importations/exportations des pays en transition.

c Pourcentage du pays dans le total des importations/exportations de tous les pays partenaires de la Suisse. 


\section{C.2.3. Cinq exemples de produits agricoles importés par la Suisse}

Le tableau suivant présente des données sur les principales provenances de quelques produits importés par la Suisse. Les importations ont beaucoup varié en valeur d'une année à l'autre dans les années 1990, notamment en raison des fortes fluctuations des prix mondiaux pour certains produits comme le café et le cacao.

Tableau 7: Principaux pays fournisseurs de certains produits agricoles importés par la Suisse, 2004 (en milliers de francs et en pourcentage)

\begin{tabular}{|c|c|c|}
\hline Roses fraîches coupées pour bouquets & en milliers de francs & en $\%$ du total \\
\hline Pays industrialisés & $32^{\prime} 142.6$ & 60.5 \\
\hline Equateur & $8 ' 351.8$ & 15.7 \\
\hline Kenya & $6 \prime 782.7$ & 12.8 \\
\hline Zimbabwe & 2'697.1 & 5.1 \\
\hline Tanzanie & 1'620.7 & 3.1 \\
\hline Colombie & 1'070.5 & 2.0 \\
\hline Divers & 440.3 & 0.8 \\
\hline Total & $53^{\prime} 105.7$ & 100.0 \\
\hline Evolution des dernières années & en milliers de francs & en $\mathrm{fr} /$ quintal \\
\hline 1990 & $21^{\prime} 044.2$ & 1748.5 \\
\hline 1995 & $288^{\prime} 067.0$ & 1779.0 \\
\hline 2000 & $58^{\prime} 371.7$ & 1687.5 \\
\hline 2003 & $57^{\prime} 732.3$ & 1515.9 \\
\hline 2004 & $53^{\prime} 105.7$ & 1415.5 \\
\hline
\end{tabular}

\begin{tabular}{lrr}
\hline Bananes & en milliers de francs & en \% du total \\
\hline Costa Rica & $41^{\prime} 617.1$ & 41.3 \\
\hline Colombie & $23^{\prime} 534.8$ & 23.3 \\
\hline Equateur & $16^{\prime} 207.1$ & 16.1 \\
\hline Rép. dominicaine & $6^{\prime} 758.7$ & 6.7 \\
\hline Panama & $5^{\prime} 548.2$ & 5.5 \\
\hline Pérou & $2 ' 323.8$ & 2.3 \\
\hline Divers pays de I'UE & $1^{\prime} 792.6$ & 1.8 \\
\hline Divers pays en développement & $3^{\prime} 057.7$ & 3.0 \\
\hline Total & $\mathbf{1 0 0} \mathbf{8} 8 \mathbf{0 . 0}$ & $\mathbf{1 0 0 . 0}$ \\
\hline Evolution des dernières années & en milliers de francs & en fr./quintal \\
\hline 1990 & $95^{\prime} 430.9$ & 125.8 \\
\hline 1995 & $88^{\prime} 961.5$ & 118.6 \\
\hline 2000 & $103^{\prime} 456.2$ & 143.0 \\
\hline 2003 & $105^{\prime} 613.0$ & 146.3 \\
\hline 2004 & $100^{\prime} 840.0$ & 137.1 \\
\hline
\end{tabular}




\begin{tabular}{lrr}
\hline Café non torréfié & en milliers de francs & en \% du total \\
\hline Brésil & $34^{\prime} 943.0$ & 25.5 \\
\hline Colombie & $16^{\prime} 736.4$ & 12.2 \\
\hline Mexique & $13^{\prime} 933.1$ & 10.2 \\
\hline Guatemala & $8^{\prime} 475.9$ & 6.2 \\
\hline Inde & $7^{\prime} 120.8$ & 5.2 \\
\hline Costa Rica & $6^{\prime} 806.1$ & 5.0 \\
\hline Ethiopie & $6^{\prime} 720.3$ & 4.9 \\
\hline Honduras & $5^{\prime} 715.5$ & 4.2 \\
\hline Ouganda & $5^{\prime} 331.5$ & 3.9 \\
\hline Kenya & $5^{\prime} 203.8$ & 3.8 \\
\hline Laos & $3^{\prime} 635.6$ & 2.7 \\
\hline Pérou & $3^{\prime} 225.3$ & 2.4 \\
\hline Indonésie & $3^{\prime} 155.1$ & 2.3 \\
\hline Autres pays & $16^{\prime} 001.9$ & 11.7 \\
\hline Total & $\mathbf{1 3 7} \mathbf{0} 04.3$ & $\mathbf{1 0 0 . 0}$ \\
\hline Evolution des dernières années & $220^{\prime} 881.8$ & 340.0 \\
\hline 1990 & $242^{\prime} 712.7$ & 422.4 \\
\hline 1995 & $232^{\prime} 748.8$ & 349.3 \\
\hline 2000 & $144^{\prime} 036.8$ & 199.4 \\
\hline 2003 & $137^{\prime} 004.3$ & 208.1 \\
\hline 2004 & & en fr./quintal \\
\hline
\end{tabular}

\begin{tabular}{lrr}
\hline Fèves de cacao & en milliers de francs & en \% du total \\
\hline Ghana & $22^{\prime} 540.3$ & 32.7 \\
\hline Equateur & $13^{\prime} 102.9$ & 19.0 \\
\hline Côte d'Ivoire & $11^{\prime} 162.7$ & 16.2 \\
\hline Grenade & $1^{\prime} 936.9$ & 2.8 \\
\hline Jamaïque & $1^{\prime} 835.1$ & 2.7 \\
\hline Nigeria & $1^{\prime} 743.9$ & 2.5 \\
\hline Venezuela & $1^{\prime} 413.7$ & 2.0 \\
\hline Cuba & $1^{\prime} 203.9$ & 1.7 \\
\hline Pays de I'UE & $12^{\prime} 358.6$ & 17.9 \\
\hline Autres pays & $1^{\prime} 719.9$ & 2.5 \\
\hline Total & $\mathbf{6 9} \mathbf{9} \mathbf{0 1 7 . 9}$ & $\mathbf{1 0 0 . 0}$ \\
\hline Evolution des dernières années & $53^{\prime}$ '482.6 & en fr./quintal \\
\hline 1990 & $50^{\prime} 757.4$ & 250.3 \\
\hline 1995 & $49^{\prime} 019.1$ & 213.5 \\
\hline 2000 & $92^{\prime} 889.6$ & 222.9 \\
\hline 2003 & $69^{\prime} 017.9$ & 351.2 \\
\hline 2004 & & 271.7 \\
\hline
\end{tabular}

\begin{tabular}{lrr}
\hline Beurre de cacao, graisse et huile & en milliers de francs & en \% du total \\
\hline Pays-Bas & $80^{\prime} 224.2$ & 75.2 \\
\hline France & $17^{\prime} 539.1$ & 16.4 \\
\hline Autres pays industrialisés & $8^{\prime} 267.9$ & 7.8 \\
\hline Pays en développement & 612.9 & 0.6 \\
\hline Total & $\mathbf{1 0 6} 6 \mathbf{6 4 . 1}$ & \\
\hline
\end{tabular}

Source: Direction générale des douanes, Commerce extérieur de la Suisse, statistique selon les marchandises et les pays, Berne, Annuaires 2004, 2000, 1995 et 1990, pour les lignes tarifaires 0603.1072 (roses), 0803.0000 (bananes), 0901.1100 (café), 1801.0000 (cacao). 


\section{C.3.1. Stock des investissements à la fin de 2003}

Le rôle des investissements directs est important pour les pays en développement: ils favorisent le transfert de technologies et la création d'emplois; en outre, moins volatils que les investissements de portefeuille, ils constituent des capitaux relativement stables pour les pays en développement.

En 2003, les stocks totaux des investissements suisses à l'étranger s'élevaient, selon les chiffres publiés par la Banque nationale suisse, à 424 milliards de francs, dont $43 \%$ investis dans des pays de l'Union européenne, $31 \%$ dans les autres pays industrialisés et $26 \%$ (112,1 milliards de francs) dans des pays en développement ou en transition (voir graphique 2).

\section{Graphique 2: Répartition des stocks d'investissements directs suisses à l'étranger, fin 2003 (en milliards de francs)}
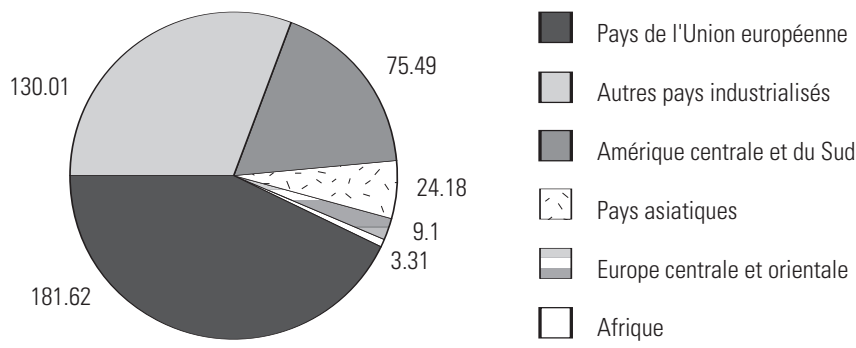

Source: Banque nationale Suisse, <www.snb.ch>, sous "Publications», "Balance des paiements et position extérieure nette de la Suisse", "Investissements directs".

Les investissements directs à l'étranger se concentrent sur un petit nombre de pays en développement ou en transition. D’autres pays et régions restent largement à l'écart des investissements. Le stock des investissements dans l'ensemble des pays africains ne représentait par exemple que 3,31 milliards de francs, soit 0,8\% des investissements totaux (ou 3\% seulement des investissements dans les pays en développement et en transition). Les investissements en Afrique se concentrent surtout sur l'Afrique du Sud (1,15 milliard), l'Egypte (338 millions) et le Maroc (263 millions de francs).

Le graphique 3 montre les pays en développement ou en transition où les stocks d'investissements sont les plus importants à la fin de 2003. 50,3\% des investissements sont concentrés dans les centres financiers offshore ${ }^{1}, 81 \%$ se sont concentrés sur ces centres offshore et les 14 pays principaux de destination des investissements suisses.

1 Centres financiers offshore: Anguilla, Bahamas, Barbade, Bermudes, îles Vierges britanniques, Jamaïque, îles Caïmans, Montserrat, Antilles néerlandaises, Panama, Saint-Kitts-et-Nevis et, depuis 2000, Antigua-et-Barbuda, Belize, Dominique, Grenade, Sainte-Lucie, Saint-Vincent-et-les-Grenadines, îles Turks et Caicos. 
Graphique 3: Principaux pays en développement et en transition de destination des investissements directs suisses, stock fin 2003 (en milliards de francs)

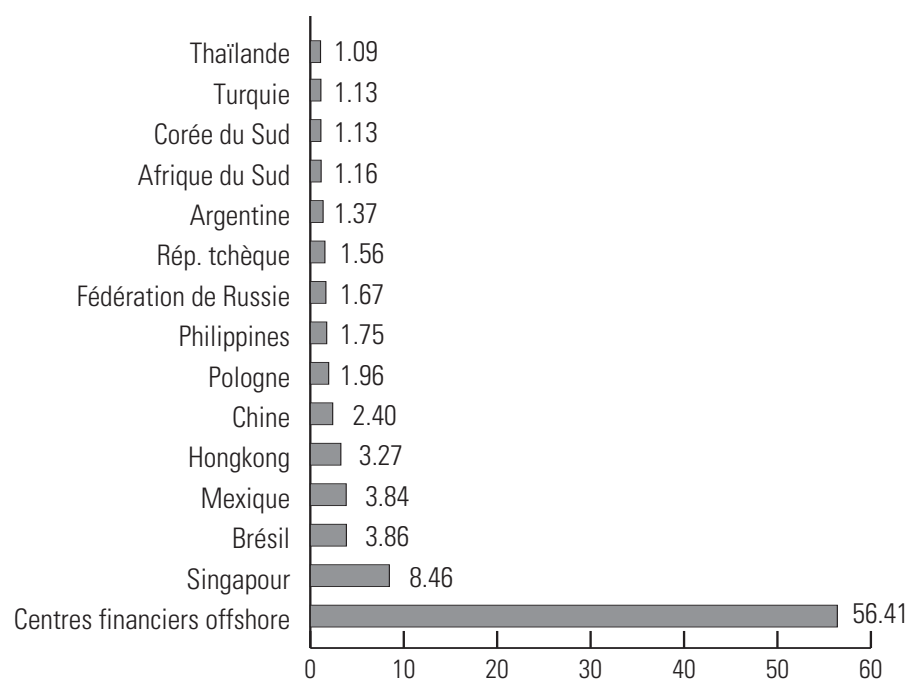

Source: Banque nationale suisse, <www.snb.ch〉, sous "Publications», "Balance des paiements et position extérieure nette de la Suisse", "Investissements directs».

\section{C.3.2. Flux d'investissements directs suisses à l'étranger en 2003}

Les statistiques de la Banque nationale suisse montrent les flux d'investissements directs suisses par pays de destination. Les flux varient beaucoup d'une année à l'autre. Ces dernières années, ils se sont élevés à 50 milliards de francs en 1999 puis à 75,4 milliards en 2000, pour baisser ensuite à 31 milliards en 2001 et 12,2 milliards en 2002 .

En 2003, les flux d'investissements directs suisses à l'étranger ont atteint 20,4 milliards de francs, dont 6 milliards destinés à des pays de l'Union européenne et 4 milliards à des pays en développement et des pays en transition. Comparés aux montants de l'APD (1,7 milliard de francs en 2003), ces investissements sont donc des flux importants. Mais il faut préciser qu'ils se dirigent vers quelques régions et pays seulement, comme le montre le graphique $4^{2}$, et que d'autres régions restent totalement à l'écart de ces flux. En 2003, ils étaient largement négatifs envers l'Afrique, marquant un désinvestissement de 248 millions de francs.

2 Le total des flux d'investissements pour les pays mentionnés dans le graphique 4 est supérieur au total des investissements totaux dans les pays en développement et en transition. Cela s'explique par le fait que les flux d'investissements sont négatifs (désinvestissements) pour beaucoup de pays en développement en 2003. 


\section{Graphique 4: Principaux pays en développement et en transition de destination des flux d'investissements directs suisses, 2003 (en millions de francs)}

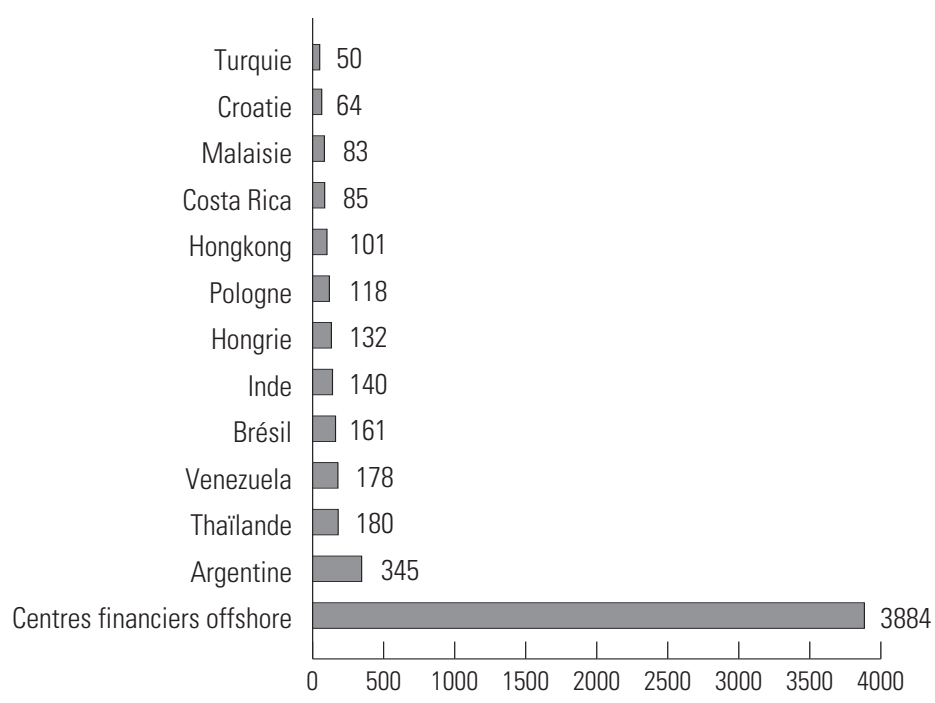

Source: Banque nationale suisse, <www.snb.ch>, sous "Publications», "Balance des paiements et position extérieure nette de la Suisse", "Investissements directs".

\section{$\square$ Précisions}

La Banque nationale suisse recueille les informations sur les investissements directs à l'étranger: lorsqu'un investisseur participe pour au moins $10 \%$ au capital d'une entreprise à l'étranger ou fonde une filiale ou une succursale à l'étranger. Les flux d'investissements enregistrés pendant une année recensent les participations aux fonds propres (fondations, acquisitions, ventes, augmentations de capital), les crédits au sein de groupes ainsi que les bénéfices réinvestis. Ce sont des flux nets, un chiffre négatif montrant un désinvestissement. Le stock à la fin d'une année plus le flux de l'année suivante n'égale pas forcément le stock à la fin de l'année suivante, car les variations de stocks proviennent aussi de facteurs qui n'apparaissent pas dans les flux (évolution du taux de change, modification dans les méthodes comptable); les acquisitions financées à l'étranger ne donnent pas lieu non plus à des flux de capitaux. Les statistiques essaient de tenir compte autant que possible du pays du bénéficiaire final, ce qui n'est pas toujours possible, comme par exemple dans le cas des centres financiers offshore. 
$\square$ Evolution de l'APD des pays du CAD et de la Suisse sur une longue période

L'APD des pays du CAD a atteint le niveau record de 69 milliards de dollars en 2003. Le précédent record en volume s'était produit en 1992, avec une aide totale de 60,9 milliards de dollars (voir graphique 5).

Exprimée en pourcentage du revenu national brut, cette hausse est cependant plus modeste, puisque l'APD des pays du CAD a passé de 0,22\% du RNB en 2001 à $0,25 \%$ en 2003, ce qui reste bien en deçà de l'objectif de $0,7 \%$ du RNB fixé par les Nations unies et même du $0,33 \%$ enregistré en moyenne pendant la période 1980-1992 (voir graphique 7).

Quelques facteurs expliquent une grande partie de l'augmentation de l'APD ces dernières années :

口 $74 \%$ de l'augmentation de l'APD de 2002 à 2003 sont dus aux effets de l'inflation et surtout de la baisse de la valeur extérieure du dollar.

$\checkmark$ Les dons bilatéraux ont beaucoup augmenté pour quelques pays particuliers, dont 1,9 milliard d'APD bilatérale en plus au seul bénéfice de l'Irak.

$\square$ Les dons nets sous forme d'annulations de dettes ont également augmenté, de 2,1 milliards de dollars. Les mesures d'allègement de dette se sont élevées à 9,4 milliards de dollars en 2003, soit près de $14 \%$ de l'APD totale des pays du CAD. Les mesures d'allègement de dette de la France ont dépassé les 2,9 milliards de dollars, soit plus de $40 \%$ de l'APD de ce pays. Pour la Suisse, ces mesures ont atteint 75 millions de dollars, soit 5,8\% de l'APD totale.

L'APD de la Suisse a également atteint un niveau record en 2003, dépassant le montant d'APD versé en 1992 lorsque la Suisse avait rejoint les institutions de Bretton Woods (voir graphique 6). Ceci s'explique en partie par un versement à l'AID différé de 2002 à 2003.

Sources: OCDE, statistiques du CAD et Commentaire de l'OCDE sur les données finales sur l'APD pour 2003, <www.oecd.org/cad>. 
Graphique 5: Evolution de l'aide des pays du CAD, 1987-2003 (en millions de dollars)

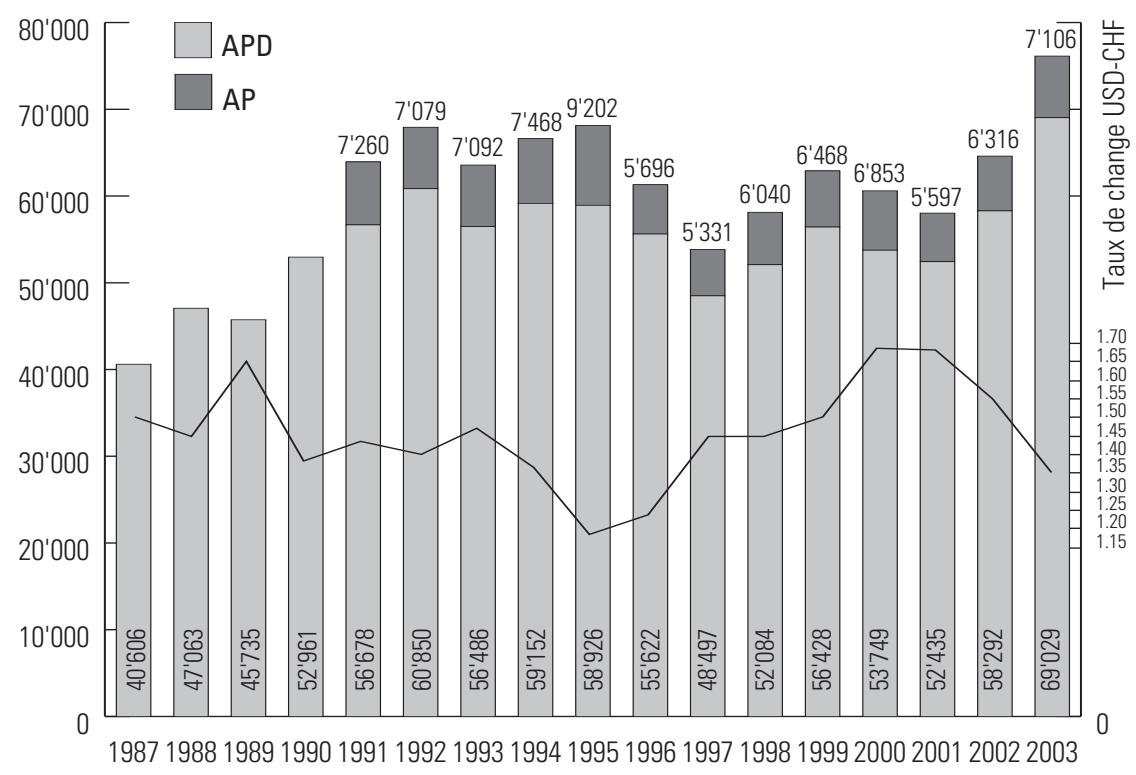

Source: OCDE, statistiques du CAD, <www.oecd.org/cad $>$.

\section{Graphique 6: Evolution de l'aide de la Suisse, 1987-2003 (en millions de francs)}

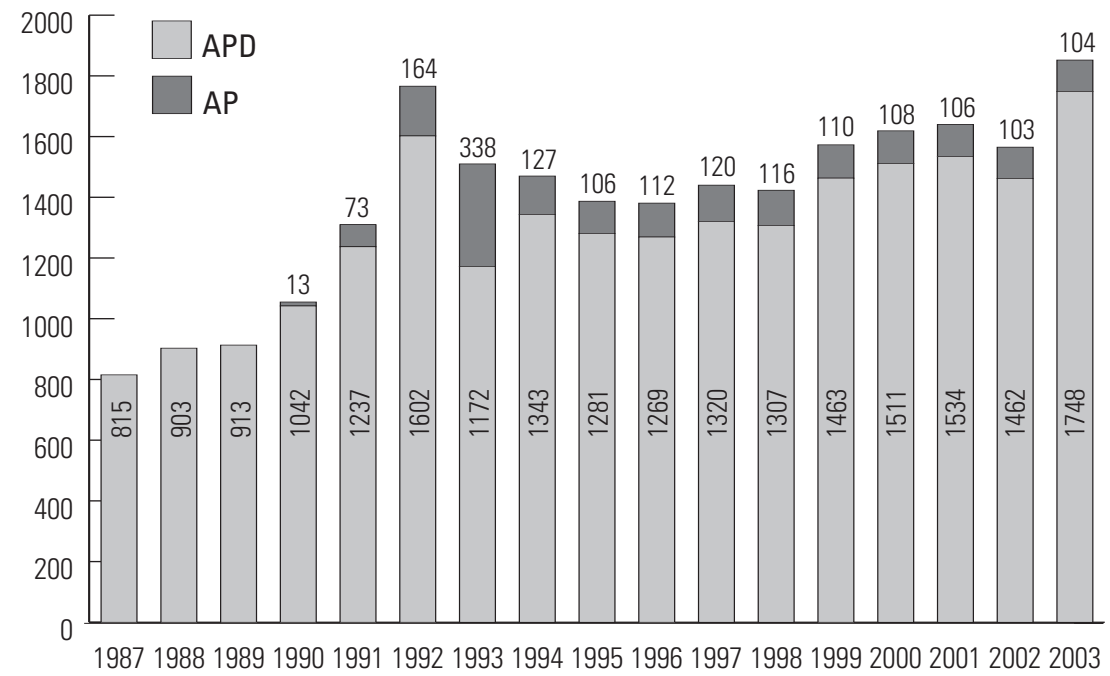

Source: OCDE, statistiques du CAD, <www.oecd.org/cad $>$. 
Graphique 7: Evolution de l'APD des pays du CAD et de la Suisse, 1986-2003 (en pourcentage du RNB)

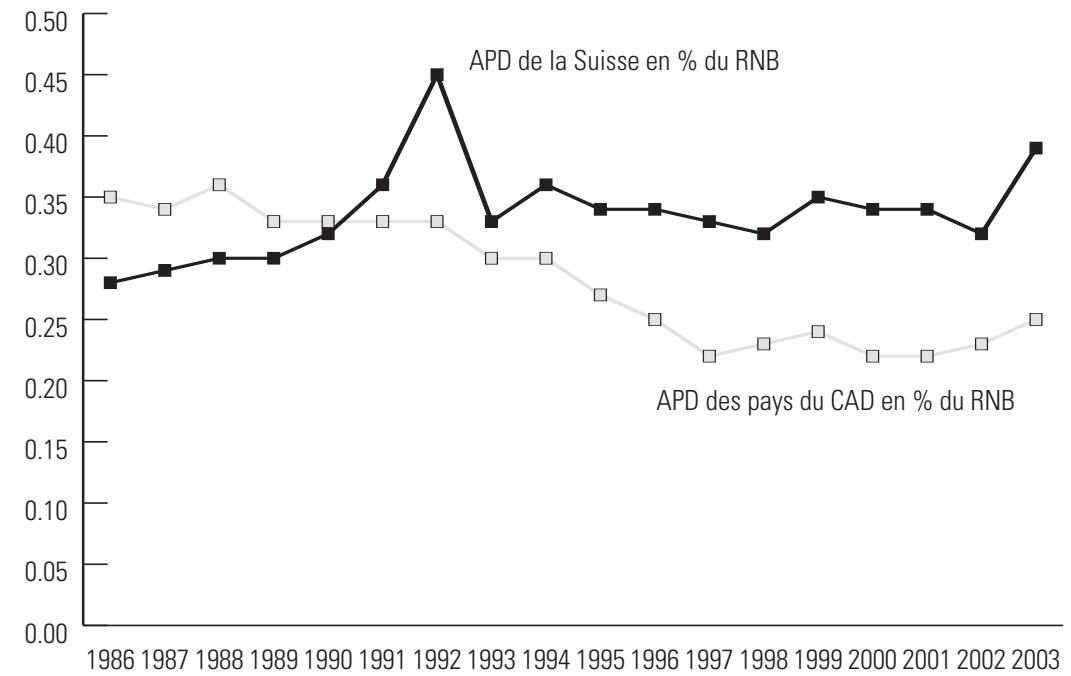

Source: OCDE, statistiques du CAD, <www.oecd.org/cad>.

L'aide des cantons et communes suisses entre dans l'agrégat d'AP ou d'APD mais est représentée dans le graphique 8 ci-dessous de manière séparée pour en constater l'évolution au cours du temps.

Les chiffres à l'origine des courbes des cantons, des communes et des ONG sont obtenus par des enquêtes annuelles, dont le taux de réponse est un élément significatif du résultat. Ils doivent donc être considérés comme des minumums.

\section{Graphique 8: Evolution de I'APD-AP et de l'aide privée des ONG pour la Suisse, 1989-2003 (index 1989 : 100)}

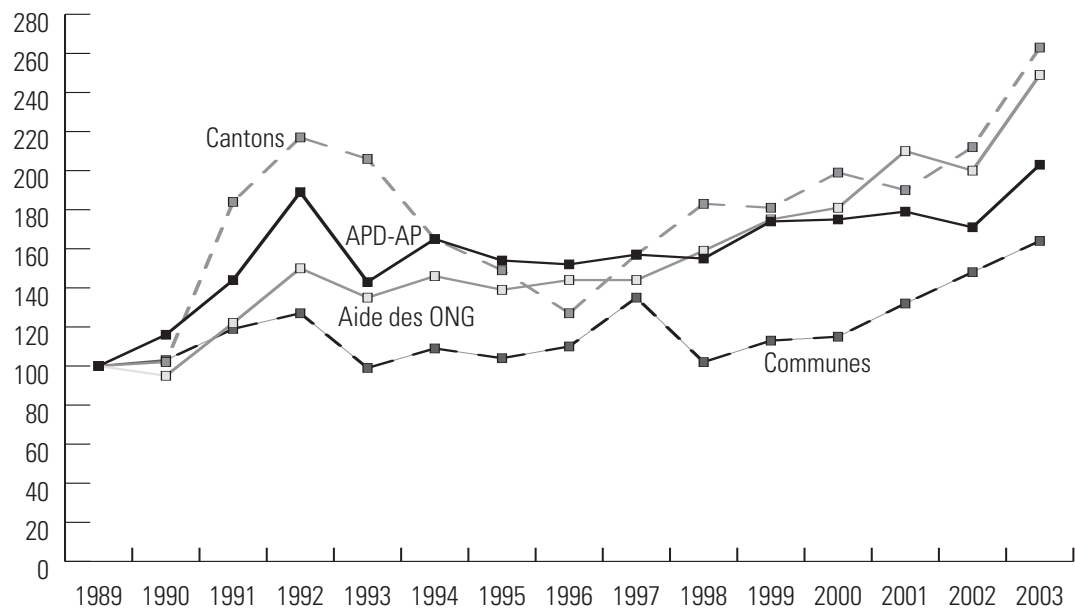

Sources: Swissaid, Aide suisse aux pays en développement, 1988-1990, Berne; iuéd/DDC, Aide suisse aux pays en développement et aux pays de l'Europe orientale, publications de 1991 à 2000, Genève; DDC, service statistique, Aide de la Suisse aux pays en développement et aux pays en transition 2001, 2002 et 2003. 
Les ONG financent des projets d'aide partiellement ou en totalité avec leurs fonds propres et les chiffres de cette aide privée se sont élevés en 2003 à 377 millions en faveur des pays en développement (partie I de la liste du CAD) et à 15 millions en faveur des pays en transition (partie II de la liste du CAD) (voir liste à la fin du présent Annuaire).

\section{Graphique 9: Evolution de l'aide privée des ONG sises en Suisse, 1986-2003 (en millions de francs)}

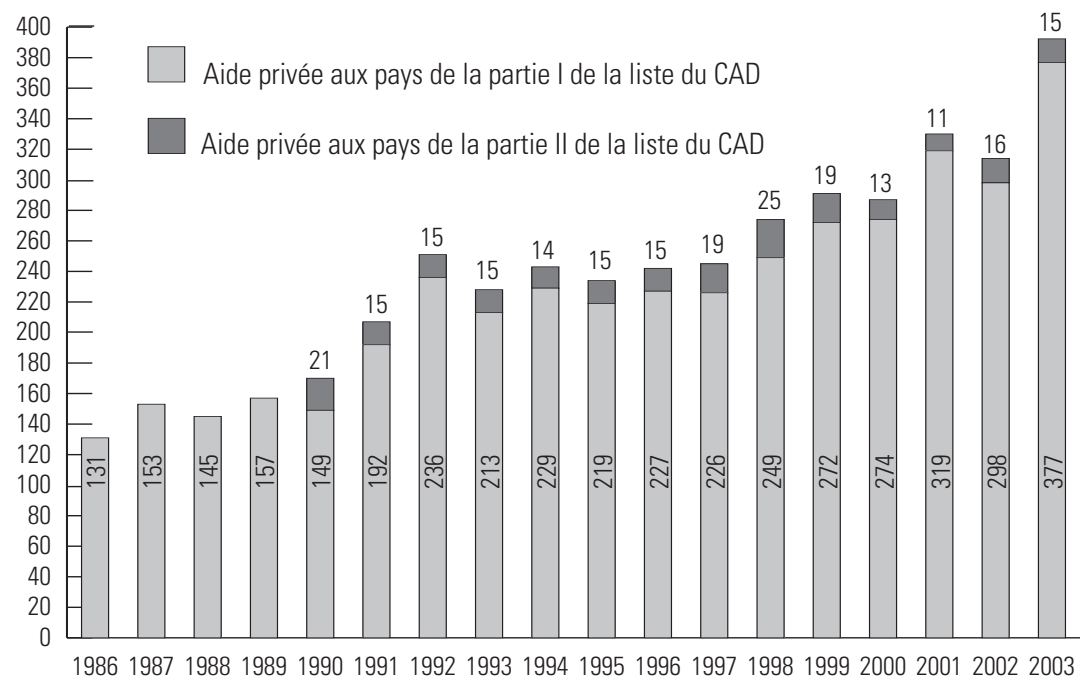

Sources: Swissaid, Aide suisse aux pays en développement, 1988-1990, Berne; iuéd/DDC, Aide suisse aux pays en développement et aux pays de l'Europe orientale, publications de 1991 à 2000, Genève; DDC, service statistique, Aide de la Suisse aux pays en développement et aux pays en transition 2001, 2002 et 2003. 